\title{
REVIEW
}

\section{A review of multiple chemical sensitivity}

\author{
R A Graveling, A Pilkington, J P K George, M P Butler, S N Tannahill
}

\begin{abstract}
Objective-To review critically the scientific literature on multiple chemical sensitivity (MCS). Definitions of MCS vary but, for this review, a broad definition of MCS was adopted as symptoms in more than one organ system elicited by various unrelated chemicals at very low levels of exposure.
\end{abstract}

Methods-A systematic literature search identified several hundred references from which key papers were selected. Two questions are considered, does MCS exist and what causes MCS.

Results and conclusions-Despite extensive literature on the existence of MCS, there is no unequivocal epidemiological evidence; quantitative exposure data are singularly lacking; and qualitative exposure data are, at best, patchy. There is also some evidence to suggest that MCS is sometimes used as an indiscriminate diagnosis for undiagnosed disorders. Despite this, the collated evidence suggests that MCS does exist although its prevalence generally seems to be exaggerated. Many causal mechanisms have been proposed, some suggesting a physical originsuch as MCS reflecting an immunological overload (total body load)—others favouring a psychological basis-such as MCS symptoms being evoked as part of a conditioned response to previous trauma. The available evidence seems most strongly to support a physical mechanism involving sensitisation of part of the midbrain known as the limbic system. However, it is increasingly being recognised that the psychological milieu of a person can considerably influence physical illness, either through generating a predisposition to disease or in the subsequent prognosis. Work is needed to establish the prevalence of MCS and to confirm or refute selected causal mechanisms.

(Occup Environ Med 1999;56:73-85)

Keywords: multiple chemical sensitivity; review

Correspondence to: Dr R A Graveling, Head of Human Sciences, Institute of Occupational Medicine, 8 Roxburgh Place, Edinburgh EH8 9SU, UK

Accepted 17 September 1998

Many authors attribute the origins of the concept of multiple chemical sensitivity (MCS) to the work of Randolph in the 1950s who went on to cofound the Society for Clinical Ecology. ${ }^{12}$ However, it has been suggested that, as a disorder, it is not entirely new. For example, Smith ${ }^{3}{ }^{4}$ suggested that Charles Darwin had what the author refers to as multiple allergy. Many theories and hypotheses have been advanced for the cause of MCS ranging from disorders of immunological function, ${ }^{56}$ to predominantly (if not entirely) a psychiatric disorder. ${ }^{78}$ Kroll-Smith and Ladd $^{9}$ present a cautionary view to be considered in assessing the validity of claims regarding MCS. They caution against a perceived tendency in the medical establishment to reject MCS as a physical disorder because it conflicts with established beliefs on medicine (so called "paradigm-induced expectations of biomedicine"), drawing on a historical perspective of the development of knowledge about the causes of disease. Clearly, in examining the scientific literature, it is important to retain an open mind. However, that should not be confused with an unquestioning mind, in which subjective assertions and rhetoric hold as much sway as scientifically designed, executed, and interpreted experimentation.

This review was commissioned by the United Kingdom Health and Safety Executive to seek answers to two questions.

- Is there convincing evidence that exposure to chemical(s), including pesticides, can result in some people in a clinical response to very low doses of that chemical or structurally unrelated chemicals?

- What is the evidence that any such reactions are a consequence of physiological processes or psychological factors?

\section{Definition of MCS}

Several phrases have been used to describe patterns of generalised hypersensitivity to chemical exposure including environmental illness; 20th century disease; chemical AIDS; and total allergy syndrome. ${ }^{10}$ However, whichever is used (and not all are exact synonyms), the disorder has not been well defined. Mooser commented on the difficulties that arose from this in seeking to examine the epidemiology of the syndrome, suggesting that: "it is quite possible that... there is not one single disorder but several different disorders with divergent underlying factors". ${ }^{11}$

Cullen $^{12}$ sought to rectify this by outlining some documentable environmental exposure(s), insult(s), or illness(es). seven major diagnostic features of the syndrome: - (1) The disorder is acquired in relation to 
- (2) Symptoms involve more than one organ system.

- (3) Symptoms recur and abate in response to predictable stimuli.

- (4) Symptoms are elicited by exposures to chemicals of diverse structural classes and toxicological modes of action.

- (5) Symptoms are elicited by exposures that are demonstrable (albeit of low level).

- (6) Exposures that elicit symptoms must be very low, by which we mean many SDs below average exposures known to cause adverse human responses.

- (7) No single widely available test of organ system function can explain the symptoms.

Cullen presented a case definition for MCS in an epidemiological context as:

"MCS is an acquired disorder characterised by recurrent symptoms referrable to multiple organ systems. These symptoms occur in response to demonstrable exposure to chemically unrelated compounds at doses far below those known to cause harmful effects in the general population. No single widely accepted test of physiologic function has been shown to correlate with symptoms". ${ }^{12}$

Ziem and Mctamney ${ }^{13}$ showed that, of 90 subjects, over $50 \%$ often reported the following symptoms (either daily or several times a week): headache, weakness, inability to concentrate, memory problems, low energy, nasal congestion, throat soreness or tightness, and joint discomfort. At least 30\% reported other symptoms affecting most body systems including: abdominal pain, nausea, visual changes, chest tightness, muscle discomfort, skin rash, sleep disturbance, and urinary disturbance. Davidoff and Keyl ${ }^{14}$ compared health complaints of people with MCS and the general population, finding that every symptom category was reported more commonly by MCS subjects, particularly central nervous system, skin, lower respiratory, and general systemic symptoms. The wide range reported and the differing pattern of symptoms between individual people make it difficult to define or establish a typical symptoms pattern for MCS.

Some authors-for example, Meggs and Cleveland ${ }^{15}$-have since published work based on the Cullen case definition. However, such definitions are not consistently or unanimously applied. For example, although Cullen differentiates between MCS and "tight building syndrome", on the grounds that the symptoms of tight building syndrome are rarely elicited away from the problem workplace, ${ }^{12}$ Hileman refers to four groups with MCS including occupants of poorly ventilated buildings, among them office workers and school children. Two of the other groups-residents of highly polluted areas and people exposed to certain consumer products, drugs, or pesticides-show the potential importance of the syndrome in that it has implications beyond occupational health extending to environmental and community health. ${ }^{16}$

Although the Cullen criteria have become extensively applied in the published literature, a broader definition was adopted for the present review. MCS was considered to be characterised by symptoms in more than one organ system elicited by various unrelated chemicals at very low levels of exposure. This broad definition was selected to avoid missing important scientific evidence. However, as will be discussed later, this may to some extent have been counterproductive in that many papers were considered that presented conflicting or contradictory evidence, possibly as a result of differing definitions for case groups. Nevertheless, on balance, the broader canvas of opinion and experimental evidence created a more complete picture of this complex and somewhat confusing collection of symptoms.

\section{Review process}

A preliminary search was conducted on four CD-ROM databases, with 12 search terms. The databases were NIOSHTIC; CISDOC; HSELINE; and EMBASE pollution and toxicology CD. This showed that most of the relevant references were obtained from the keywords "multiple chemical sensitiv", "chemical sensitiv" and "environmental illness". Other conditions such as sick building syndrome, chronic fatigue syndrome, and gulf war syndrome have symptoms which overlap with MCS. These were considered to be outside the remit of this review and were not included.

A full literature search was then conducted on all available bibliographic databases, with online versions being used to ensure that the references obtained would be as recent as possible. As well as those already referred to, these included: Psyclit; Toxline; Medline; Excerpta Medica; Chemtox; Enviroline; Environmental Bibliography; Biosis; Medical Toxicology and Health; and Pollution Abstracts as well as thesis databases and citation indices for key authors. Database abstracts were examined to select the most relevant full texts. When no abstract was available, or if it was deficient, then a conservative strategy of obtaining the full text was adopted.

Full papers were scanned for suitability for review. As a control measure, a small sample was examined independently by a second reviewer (blind to the initial assessment). The degree of concordance was $>90 \%$ and when there was a difference of opinion, the paper was included. Not all texts reviewed have been cited.

Finally, personal contacts, some arising from press coverage, provided information. Some of this was derived from research groups and what could be regarded as pressure groups in the United Kingdom and United States. Other material was obtained from individual people, notably people with MCS or with a personal interest in this subject. The contribution of these numerous groups and people is hereby acknowledged.

\section{Can clinical symptoms result from very low dose exposure to structurally unrelated chemicals?}

There is a very wide range of estimates for the prevalence of MCS among the general population. At one extreme, Cullen et al state that, at least at a level prominent enough to cause referral to an occupational and environmental 
medicine clinic, suggestions that MCS may affect $\geqslant 1 \%$ of the entire population "seem to be substantial overestimates". ${ }^{17}$ By contrast, Ashford and Miller cite several questionnaire based studies relating to general chemical sensitivity rather than specifically MCS that report that about one third responded positively to a question on perceived "special sensitivity" to chemicals. ${ }^{18}$ Although sample sizes are indicated, no participation rates are cited. Also, as indicated by the authors, it cannot be determined how many (if any) of these may develop MCS. Even in the United States therefore, where this problem has been most extensively studied, no estimate of the prevalence of MCS or any other indication of the magnitude of the problem exists. No estimates, reliable or otherwise, exist for United Kingdom or other European populations.

On the basis of published data, including case studies and written and verbal personal communications with people who apparently have MCS, there is evidence to suggest that in some people chemical exposure can result in a clinical response to very low doses of that chemical or structurally unrelated chemicals. As well as individual case histories, many papers have been published either reporting collections of case studies, ${ }^{19}$ or reports of more extensive groups of patients. For example, one of the few European studies refers to a set of 30 patients identified over a 4 year period. ${ }^{20}$

Whether this evidence is convincing depends on the criteria by which an illness is diagnosed. If an illness can only be said to exist when consistent results are obtained from well validated objective investigations, then at present such confirmatory data are lacking for MCS. This assumes however that an appropriate test battery is currently available. In both physical and psychological diseases, particularly those affecting the central nervous system, a diagnosis is often established on the basis of a consistent symptom pattern and observed changes in the functional capacity of the person. If these less stringent criteria are adhered to, then there is convincing evidence that some people report a consistent set of symptoms which they attribute to previous chemical exposure and which recur on subsequent exposure to the same or structurally unrelated chemicals. Although it is recognised that the motivation and rewards for illness are many and varied, the evidence is, at least in part, made more convincing in cases where the illness has produced considerable social and financial hardship for the person with no apparent benefit and is associated with a strong motivation to be made well.

The evidence for MCS is weakened by several other factors within the available data. MCS has been used as an umbrella term, both by individual people and medical practitioners, resulting in misdiagnosis of other medical conditions. Clinical ecologists who specialise in diagnosing environmental illness are often open to this criticism from other authors. ${ }^{21}$ As an example, a man in his 60 s attributed his prostate problems leading to surgery to MCS. ${ }^{22}$ However, development of prostatic hyperplasia is an almost universal phenomenon in aging men, with around $10 \%$ of men requiring surgery at some time, ${ }^{23}$ suggesting that this aspect at least of the patients' array of adverse health effects may have been coincidental.

There are often limited qualitative or quantitative data on chemical exposure both relative to an initiating event and subsequent trigger episodes. The incidence of a initiating event within current published data varies widely. For example, Miller and Ashford report a survey of 6800 people claiming to be chemically sensitive, of whom $80 \%$ asserted that they knew when, where, with what, and how they were made ill. Of these, $60 \%$ blamed exposure to pesticides. ${ }^{24}$ By contrast, Mooser reports a study by Rea in which 58/100 people were unable to identify a triggering set of circumstances. ${ }^{11}$ There are some documented epidemic outbreaks - for example, allegedly as a result of pesticide exposure ${ }^{25}$; and after the introduction of a new chemical plastic process. ${ }^{26}$ Paradoxically, Cullen et al found that among a group of patients with MCS at their clinic, those industrial sectors responsible for most other chemical and physical injuries were underrepresented. ${ }^{17}$ This finding has also been reported by other groups. ${ }^{27}$ Certainly the evidence does not seem to support a significant precipitating exposure to chemicals as a necessary defining prerequisite.

There are also poor exposure data on specific chemicals and the doses required to provoke symptoms. Many studies are based on self report or highly subjective test procedures. Some authors have reported considerable success with controlled challenge tests. For example, Rea et al refer to "numerous studies (several double blind)" and inhalation challenge tests on over 3000 patients with $>99 \%$ accuracy. ${ }^{6}$ However, the claims do not necessarily relate to patients with MCS. By contrast, Staudenmayer et al reported generally poor results from well controlled double blind provocation challenge tests on a group of 20 people. ${ }^{28}$ However, the criteria for inclusion of these patients are not fully documented, nor is the extent to which a diagnosis of MCS was justified. Indeed, in reporting a study of a larger group of patients, possibly including the challenge group, the same team specifically stated that the subjects did not meet the Cullen criteria, although they would have met the less stringent criteria of this review. ${ }^{8}$ There is clearly a very strong belief in a relation between chemical exposures and the occurrence of symptoms among people with MCS although objective evidence is often lacking. Due to the lack of reliable exposure data, it is also not possible to determine whether, after the response to an initiating stimulus, there is a subsequent lowering of the threshold at which further triggering stimuli elicit a similar response.

Because of the lack of good exposure data in currently published studies it is not therefore feasible to construct exposure-response relations. However, an association between exposure and disease is often accepted where a clear exposure-response relation is lacking, and consideration should also be given to whether the 
disease process is biologically plausible on the basis of exposure and outcome. The next section explores current theories for MCS.

Can a causal mechanism be established?

Many theories have been proposed to account for MCS, some biological, some psychological, and some a combination of both. Most studies have deficiencies which restrict the projection of the results to a wider population if not calling the whole study into question. Some differences between papers can probably be accounted for by differences in sample selection; the population from which they were drawn; or the defining criteria. Others are less easily accounted for.

\section{PHYSIOLOGICAL THEORIES}

In this section there is overlap between biological or physiological models and those with a corresponding psychological component.

\section{Immunological deficits}

This is one of the most commonly held theories, at least among those who subscribe to a biological explanation. In the United States it is particularly supported by those physicians known as clinical ecologists, a group often scorned in the mainstream scientific and medical literature. ${ }^{29-31}$ Most of the main papers promoting such theories present collations of clinical test results rather than the findings from experimental studies. Results are often reported for comprehensive batteries of tests of immunological function - such as white blood cell, lymphocyte and $\mathrm{T}$ cell counts; serum complement and immunoglobulin fractions; and immunophenotypic evaluations. Substantive explanatory mechanisms are seldom presented, any deviations from a normal pattern either being seen as indicative of largely unexplained deficiencies or defects in the immune system, or resulting from concepts-such as total body burden (chemical overload) - a theory often put forward by clinical ecologists or environmental physicians to account for MCS or chemical allergies as part of the immunological response. ${ }^{6}$

Although individual patients do have abnormal concentrations of some variables, no papers have been found which show a consistent pattern of abnormality indicative of a specific immunological deficit. As stated earlier, Rea et al referred to the extensive work of their Environmental Health Centre, with $>5000$ tests of immune variables (not necessarily 5000 patients). ${ }^{6}$ This work is often cited in support of an immunological theory for MCS. However, although undoubtedly related to chemical sensitivity (Levin and Byers refer to chemically induced immune dysregulation as a "recognised medical disorder" $)$ it is not necessarily applicable to MCS. Indeed, Rea et al themselves, in a relatively early work, conceded that the involvement of the immune system is not clear, stating that "involvement of the immune system, although not readily apparent, is possible". ${ }^{32}$ In a more recent work, relations between immunological function and exposure to pesticides are reported. ${ }^{33}$ However, it is poorly presented with only limited details of the patient group and serious deficiencies in analysis (acknowledged by the journal editors). Even with these serious shortcomings, the results are equivocal with no clear pattern of immunodeficiency emerging. Albright and Goldstein reported a brief review of evidence for an immunological basis for MCS including the work of Rea et $a l^{34}$ The authors cite conflicting studies, concluding that there is a lack of convincing evidence for MCS stemming from an impairment of the immune system or immunological hyperreactivity. The authors also question the concept of chemical overload (total body burden). Terr published a review of immunological theories of the causes of MCS, concluding categorically that there is no evidence that the immune system is involved. ${ }^{35}$ Similarly, Wolf stated that "none of the theories which hold immunological or allergic phenomena responsible for the development of MCS could be proved", concluding that there was a very low probability for such a causative pathway. ${ }^{36}$ Recently, Kipen et al published a small scale evaluation of 11 chemically sensitive patients. ${ }^{37}$ The authors refer to the work of Levin and Byers as "asserting that MCS was a disorder of immune regulation" although this is less apparent in the original source where much of the work reported relates to patient groups with specific chemical sensitivities. Kipen et al are rightly cautious about drawing conclusions from such a small study group although they do favour the idea that patients with MCS tend to have different immune patterns. However, the results are not consistent and conflict with some of their earlier work.

In conclusion, no consistent pattern of immune deficiency or other dysfunction can be identified among patients with MCS, even when case definition is reasonably rigorous. Some positive findings may be attributable to people with a specific chemical sensitivity (for example, an allergic antibody response to a specific substance). Some proponents of such theories contend that this variable pattern is attributable to various factors including total body burden, adaptation, and biochemical individuality. ${ }^{6}$ However, at present, the lack of consistency in response patterns both between and within individual patients with MCS limits the plausibility of this theory.

Respiratory disorder or neurogenic inflammation The heightened sensitivity to smells, often reported by patients with MCS has led some researchers to investigate this and other aspects of respiratory function for possible causative mechanisms. Doty et al reported the results of an experimental study in which measures of olfactory sensitivity, respiration rate, and nasal airflow resistance were compared in patients with MCS and normal controls. ${ }^{38}$ Not all of the MCS group necessarily met the review criteria for MCS as two did not show multisystem symptoms. The expected difference in odour threshold did not emerge, although patients with MCS did show significantly higher nasal resistances before and after testing and respiration rate was also significantly higher. The authors draw inferences about a localised allergic 
reaction, although this offers little explanation for the wider scale of reactivity shown by patients with MCS.

Bascom briefly reviewed the evidence for MCS being related to altered function of the respiratory mucosa, ${ }^{39}$ suggesting that chemicals stimulate a non-specific pathway involving c-fibre neurons; these are unmyelinated nerve fibres widely distributed in the respiratory mucosa (nose to lower airways). Stimulation of the fibres in animals results in local release of neuropeptides which can cause dilatation or constriction of blood vessels, and airways. It is hypothesised that differential responsiveness to tobacco smoke reflects differential function of c-fibre neurons in humans. The respiratory mucosa is also able to release several immunoregulatory substances such as cytokines (acute phase response to irritants), which act locally. Although it is feasible that the respiratory mucosa could act as both a target and effector organ for MCS, this hypothesis alone does not account for the wider multisystem symptoms reported in MCS.

The possible role of c-fibres and respiratory mediated inflammation is discussed further by Meggs et al in a series of papers. Meggs and Cleveland described a group of 10 patients with MCS, of whom nine had experienced prominent nasal symptoms. ${ }^{15}$ Nasal examination and rhinolaryngoscopy were abnormal in all 10 cases. The authors suggested that they could have a form of reactive airways dysfunction syndrome (RADS) affecting the upper airways (hence RUDS - reactive upper airways dysfunction syndrome). So RADS is a chronic asthma-like condition developing after an acute irritant exposure, although RUDS is chronic rhinitis developing in temporal association to inhalation exposure to a toxic substance.

The authors invoke neurogenic inflammation to account for these symptoms, but acknowledge that the symptoms of patients with MCS are not restricted to the upper airway (and offer no hypothesis to account for this). Neurogenic inflammation is the focus of a further paper, ${ }^{40}$ providing a link with the work by Bascom in referring to c-fibres as the locus for such inflammation. Neurogenic inflammation is the mechanism by which substances released from sensory nerve endings - such as substance $\mathrm{P}$-produce an inflammatory response. The author describes the neurochemical pathways for neurogenic inflammation and then briefly cites claims for an involvement of such inflammation in several disorders including migraine headaches, and less strongly, in rheumatoid arthritis and fibromyalgia. It is suggested that other systems may be affected by either of two mechanisms (or both). The first is the release of interleukins, which can influence central nervous activity (despite the report that they normally only acted locally) ${ }^{39}$ The second invokes the concept of neural switching: a form of cross over between nerve pathways.

Meggs suggests studies examining concentrations of two substances, neural endopeptidase (NEP) and substance $\mathrm{P}$ as a means of testing the neurogenic hypothesis. Meggs et al report such a study in patients with RUDS. ${ }^{41}$ Although not technically diagnosed as having MCS, an overlap is suggested between the conditions as the patients studied would meet most case definitions for MCS. Although all patients had extensive abnormalities of the upper airways, examinations for substance $P$ and NEP were not helpful because of a high general level of uptake of the tissue stain (nonspecific staining). In a later paper, Meggs returns to the idea of neural or neurogenic switching. ${ }^{42}$ The author cites examples of symptoms between systems - such as respiratory symptoms or urticaria resulting from food allergies or "gustatory rhinitis" with respiratory symptoms and facial sweating after the ingestion of spicy foods. However, according to Sparks et al, all of the experimental data on which these hypotheses are based are derived solely from patients with MCS or RUDS with no controlled studies of objective signs of inflammation of the upper airways. ${ }^{21}$ These authors briefly refer to neurogenic inflammation and interleukin transfer suggesting that neurogenic inflammation might help to explain some multiorgan system complaints and commenting on an animal study which apparently provides a degree of support. However, transmission of effect to another organ system has been described by Miller and Ashford relative to the cardiovascular reflex response to noxious chemicals. ${ }^{24}$ Trigeminal free nerve endings in the nose and throat trigger a protective pattern of responses including reduction in heart rate and cardiac output. Thus the pattern described earlier may be an acute reflex response rather than an indication of any more chronic or insidious defect.

In conclusion, although there is some evidence of nasal or upper airway involvement in at least some groups of patients with MCS, these symptoms alone cannot account for the multisystem pathology. Theories have been proposed which seek to explain transmission of effects to other organ systems, but at this time, no direct experimental support from patients with MCS has been identified and there is only limited support from animal studies.

Olfactory-limbic system, kindling and sensitisation models

The proponents of these theories suggest that the interconnections between the olfactory nerve (sense of smell), the brain limbic system (considered to be the centre of the emotions) and the hypothalamus (controls organ function by hormone release) may be important when chemicals trigger a multisystem response. The limbic system comprises several elements including the amygdala and hippocampus. It has been shown in animal studies that the amygdala is particularly vulnerable to sensitisation, the process by which repeated exposure to a specific agent results in an increased response by the organism at doses lower than those normally expected to elicit any response. It is suggested that neural sensitisation can occur by both kindling and non-kindling mechanisms. Kindling is a process by which low level 
electrical or chemical stimuli, which initially have no effect on behaviour, eventually elicit persistent susceptibility to convulsive seizures. Time dependent sensitisation, considered to be a non-kindling type of neural sensitisation, is the progressive amplification of response to repeated intermittent exposure to a chemical or non-chemical stimulus. Animal studies have shown that time dependent sensitisation shares several features with MCS including cross sensitisation to differing chemicals. ${ }^{43}$ Both mechanisms would in theory be capable of eliciting a multisystem response through the hypothalamus and associated structures.

The putative association between MCS and the limbic system is widely attributed to Bell some 20 years ago. In 1992, Bell et al reviewed and developed the concept of limbic kindling, described as a special type of time dependent sensitisation of olfactory-limbic neurons. ${ }^{44}$ They describe the different stages in a mechanism initiated by chemicals entering the central nervous system through the olfactory system, which lacks the blood-brain barrier found in other parts of the central nervous system. Studies on rats are cited to show that substances can migrate from sensory neurons in the nose to the olfactory bulb and thence to other areas of the brain. Unsupported by human or even higher animal studies, this pathway could provide an explanatory mechanism for the spreading of sensitisation to a range of unrelated somatic systems. However, the authors themselves state that, although the paper offers an argument for the possibility of kindling, no systematic data on kindling in patients with MCS have been collected to test this.

Ashford and Miller outline several human and animal studies relating structures of the limbic system to behavioural patterns, illustrating how, once chemicals have gained access to the system, the various parts of the system have the capability to influence behaviour and other responses in a manner consistent with some patients with MCS. ${ }^{18}$ Further studies, again supporting the idea of a link between limbic kindling and MCS, were described by Adamec who reported that kindling had been produced in many species including primates and possibly humans. ${ }^{45}$ This paper emphasises the relation between chemically induced kindling and anxiety, an important relation as anxiety is often a feature of patients with MCS.

Bell et al use the concept of time dependent sensitisation in relation to cacosmia (chemical odour intolerance), a disorder related to MCS. ${ }^{46}$ They describe a study of self reported illness, which they consider to provide useful confirmatory evidence for a role for opioid or dopaminergic involvement with time dependent sensitisation relative to the action of excitatory amino acids on limbic pathways and the hypothalamo-pituitary-adrenal axis. The obvious limitation of the data being self reported is acknowledged, although the consistency of the data with other previous work is considered to support the findings. A further related hypothesis was proposed by Corrigan et al who reported on five case studies of people with fatigue syndromes similar to $\mathrm{MCS}^{47}$ The authors suggest that the symptoms are consistent with altered sensitivity of $\gamma$-aminobutryric acid (GABAa) mediated pathways in the limbic system after exposure to insecticides or pesticides (GABAa is an excitatory amino acid). The exposure information is, however, entirely anecdotal, and limited evidence is cited to support the hypothesis in the form of evidence of a GABAa mediated effect of other pesticides.

Some support for a central neural effect of pesticides and solvents can be obtained from the work reported by Heuser et al in which quantitative and qualitative studies of regional cerebral blood flow in patients thought to have been exposed to these chemicals showed deficiencies in comparison with normal controls. ${ }^{48}$ The pattern of deficit differed from that recorded for patients with depressive illness or chronic fatigue syndrome. Apart from the limitations of the exposure information there is no indication that the patients could be classified as having MCS. Also, the locus of the deficiency is equivocal, as the pattern was more consistently that of direct cortical deficiency, with temporal (limbic) involvement only significant in the group of younger patients. Nevertheless, the paper does offer limited support to the potential for such injury relative to two classes of chemicals often associated with MCS and also provides an avenue for further objective appraisal of patients with MCS.

A review by Lorig et al seems to indicate that subthreshold odours can have physiological and behavioural effects without their presence being recognised. ${ }^{49}$ For example, subthreshold odours were shown to produce different electroencephalographic patterns. The authors suggest that low exposures may affect central nervous system activity. These findings, in normal subjects, do support a biological explanation for MCS in indicating the potential for undetectable concentrations of chemicals to exert measurable effects on the body.

More recently, Bell et al have published the results of a study which seems to show some support for a neural mechanism for MCS. The first paper reports the results of an extensive study of college students based on a questionnaire about odour sensitivity (cacosmia) and noise sensitivity. ${ }^{50}$ The authors hypothesised that those who were more sensitive to both might show more indication of limbic dysfunction or other characteristics which could be related to MCS. Measures included the use of a limbic system checklist, designed to assess the various dimensions of ictal limbic dysfunction associated with temporal lobe epilepsy. Although the students who reported high frequency of illness from environmental chemicals scored higher on all four subscales (somatic, sensory, memory, behavioural, $\mathrm{p}<0.0001)$ these did not differentiate between those with high cacosmic scores alone and those with both high cacosmia and high noise sensitivity. There were some differences in other characteristics, notably in relation to alcohol and smoking, together with anxiety and the Barsky amplification scale (assessing the intensity of experiencing environmental and 
somatic changes and personality traits suggesting shyness). The authors acknowledge the limitations imposed by the use of subjective ratings, but they defend their use. They also indicate that many of the significant differences are not large and were unlikely to be clinically important. Nevertheless, the findings from a group of basically healthy people are seen as supportive of limbic system theories.

This work is among that cited in a second paper which reviews the evidence for olfactory limbic neural sensitisation as a model for MCS. ${ }^{51}$ The author cites extensively, particularly from the work of her own group, to support this concept, presenting evidence considered to provide face validity; construct validity; and predictive validity. One major limitation in both these pieces of work, is that the evidence is derived exclusively from carcosmic people. Although having the advantage of a healthy study population there is a clear need for work on a clinically more appropriate population.

This paper indicates that these mechanisms can be initiated by psychological (stress) stimuli as well as chemical agents. On first consideration, this presents a potential conflict in distinguishing between psychologically mediated kindling, centring on the limbic system, and the alternative explanation of psychological conditioning, in which the central neural focus is again part of the limbic system (the amygdala)..$^{52}$ In a very recent publication, Bell et al consider this specific topic, documenting differences in the patterns of response between conditioning and sensitisation, relative to the response to both the initial and subsequent stimulation..$^{53}$ Although the pattern is to some extent dependent on the context of exposure, it does seem that these mechanisms involve different pathways and can be differentiated. This is discussed further in the next section, on psychological theories. However, the authors also suggest that panic disorders reported by a percentage of subjects with MCS may be a manifestation of kindling, with a subset showing partial seizure activity and that nonkindling sensitisation could be the result of modulation of dopamine pathways in the olfactory limbic system. Dopamine is involved in odour discrimination and also modifies the response to stress (through release of prolactin). The implication is that repeated low level chemical exposures can modify response to stressors in some people. In a second recent paper, Bell coauthored a report of a working group relating to sensitisation and kindling which briefly documents the main features of sensitisation and kindling. ${ }^{54}$ The authors outline an experimental plan, involving both cross sectional (case-control) and longitudinal studies on humans, together with a series of animal studies, which would test the neural sensitisation hypothesis. Although this would be done through animal studies, it should provide further information on the underlying mechanisms. The authors also refer to an experimental procedure which would differentiate between sensitisation and conditioning, thereby eliminating conditioned responses as the causal mechanism.

Sensitisation (through kindling and nonkindling mechanisms) is a well reasoned and plausible theory to account for MCS. The main proponents, particularly Bell, have assembled an effective array of indirect supportive evidence from a mixture of studies on animals and on humans with related problems. (Evidence from cacosmic people suggests that there are several people with inborn or acquired increased capacity to sensitise to the environment. This subgroup also show higher scores for limbic dysfunction, the system implicated in the sensitisation process. There are no confirmatory physiological data on this group.)

Although no direct evidence from human populations has yet been produced, it is interesting to note that, unlike other explanatory theories, no papers have been identified attempting to refute this explanation. The theory therefore remains as yet unproved with no apparent serious detractors. It must therefore stand as an untested but tenable theory based on a neurophysiological mechanism.

Toxicant induced loss of tolerance (TILT)

Very recently, Miller has published a detailed exposition of an explanatory theory which seeks to explain MCS in toxicological terms while accounting for the fact that responses are apparently provoked at exposure levels well below those ordinarily regarded as toxic. ${ }^{55}$ The author presents this as a new theory of disease, drawing parallels with germ theories and immune theories to illustrate the point. Not least among these parallels is the initial antagonism with which previous disease theories have been met by the established medical profession.

The theory involves two phases: an initial loss of tolerance caused by chemical exposure, followed by apparently disproportionate responses to exposure to other substances. No mechanism is identified or postulated to account for the initial loss other than "possibly but not necessarily due to sensitisation". It is suggested that specific toxicants produce a specific pattern of responses, although this may be masked by responses to other exposures still affecting the subject. Anecdotal evidence suggests that some patients with apparent MCS can relate specific patterns of symptoms to exposure to particular chemicals or groups of chemicals, which would support this. The author draws parallels with the established pattern of responses to drug addiction, differentiating between the two phenomena by the statement that the responses of chemically sensitive patients are not primarily to drugs. This seems to be a somewhat arbitrary distinction in view of variations in what are regarded as drugs in different societies. It is not immediately clear how the TILT symptoms differ from classic addictive behaviour theories. However, there is a clear implication of some form of individual susceptibility or intolerance as a contributory element. 
It is suggested that, if a subject is exposed to test substances in an environmental chamber before they have been cleared of these masking responses, false responses may be evoked as the subject is unable to differentiate new symptoms from those associated with the previous exposure. The author uses the analogy of testing for caffeine intolerance, where it is desirable to control exposure for a period before testing to allow withdrawal symptoms to subside to avoid erroneous responses.

The paper lays out a plausible and testable explanation of the theory, at least as far as consequent heightened sensitivity or hypersensitivity. An accompanying paper describes the research methods that could be adopted. ${ }^{56}$ The description of how residual effects could account for erroneous and misleading responses in testing exposure seems reasonable albeit as yet largely theoretical. Much of the supporting material is drawn from studies on other populations-such as those with addictions-or is essentially anecdotal. However, the main deficiency of the paper lies in its failure to consider the causal mechanism. The text is almost entirely concerned with the development of symptoms of MCS with only the brief reference to cause already mentioned, although some general reference is made to the role of individual susceptibility.

Without an adequate explanation for the cause of the initial loss of tolerance, the theory explains the pattern of symptoms, not the disease and, although the pattern of symptom response to a particular challenge might be consistent, no explanation is given to account for the apparent spread of sensitivity to other unrelated chemical groups.

Porphyria

Disturbances in the production of haemoglobin are characteristic of porphyrias, which some have implicated in the aetiology of MCS. Although some forms of porphyria are inherited, chemical exposure can cause or at least trigger disorders in haem synthesis. However, these normally occur with relatively high exposures and most of the evidence is drawn from animal studies. ${ }^{57}$ It has been reported that over $85 \%$ of those with MCS, also have porphyria disorders. ${ }^{58}$ This seems an overestimate as in the United Kingdom porphyria is a relatively rare condition affecting only 1 in 50000 people. Only one published text is cited in support of this. ${ }^{13} \mathrm{~A}$ table in this publication shows 12 out of 14 patients $(85.7 \%)$ with at least one metabolic test result outside the cited normal range. However, there are several deficiencies with this interpretation. Firstly, although the values cited are outside the stated normal range they are not necessarily within the cited clinically abnormal range as there was often a gap between these two ranges. A more serious shortcoming, however, is the unrepresentativeness of the patients tested, as only those with symptoms suggesting porphyrin disorders were tested. The inference drawn should be that $85 \%$ of those patients with MCS reporting symptoms of porphyrin disorders showed biochemical markers for such disorders outside the normal range. No value is given for the number of patients seen at the clinic during that period and it is difficult to establish this from the paper as the timescales for the tests conducted differed. Results for up to 92 patients are reported for a questionnaire, introduced in the late 1980s. By contrast, results are reported for 68 patients tested for immune function until the end of March 1994, which are described as not the same patients as a set of 23 tested from April 1994.

Daniell et al incorporate brief reference to the data from this paper as a conference paper awaiting publication. ${ }^{57}$ It is not clear therefore to what extent these data were included in their deliberations. The authors express some concerns with the limitations of laboratory test procedures for porphyria indicators and refer to a general lack of cutaneous symptoms among patients with MCS. They conclude that there is no convincing evidence that there is - or is not - any such increased prevalence of abnormal measures of haem synthesis associated with MCS syndrome. They state that the proposed relation should, at most, be considered speculative and unestablished, as those papers which report such relations have methodological deficiencies which undermine their validity.

\section{PSYCHOLOGICAL THEORIES}

\section{Conditioned response}

Classic Pavlovian conditioning offers a mechanism whereby somatic responses can be produced in response to apparently inappropriate stimuli. As stated earlier, some authors have suggested that the responses of patients with MCS may fall into this category, particularly where traumatic chemical exposure has occurred. In this case, it would be expected that the incidence of MCS would be higher among those more likely to experience such exposures, although the evidence of some authors does not support this. ${ }^{17}$

A specific example, likely to provoke controversy, is the report of an apparently very high incidence of childhood physical or sexual abuse, identified during psychotherapy. Staudenmayer et al report the results of a study which suggested that $60 \%$ of their patient group reported childhood sexual abuse and that psychotherapy alleviated symptoms of chemical sensitivity. ${ }^{8}$ The authors interpret this as a form of conditioning, with odours associated with the trauma from childhood initiating a physical response. The reported work has some methodological deficiencies-such as an incomplete description of the selection procedures for patients and no systematic examination of any relation between the conditioning stimulus and the pattern of somatic responses. Nevertheless, it supports an association between abuse or trauma and MCS, referring to several other studies where ill defined somatic symptoms or disorders are associated with previous abuse. Alternatively, Pennebaker briefly refers to research indicating a tendency for the victims of trauma to be more likely to report minor health problems, suggesting that this may be part of a defence mechanism, ${ }^{59}$ a theory refuted 
by Staudenmayer et al on the basis of the success of psychotherapy, or part of the long term biological stress response to the trauma. Although several authors have referred to conditioning either as a causal or contributory factor, ${ }^{21}{ }^{36}{ }^{60}$ no studies setting out a detailed case for conditioning have been identified. Bell et al acknowledge that conditioning and sensitisation can occur together if the initiating and eliciting stimuli are given in the same physical environment..$^{53}$ Animal studies have shown that it is possible to eliminate the conditioned response to that environment by sham exposures, without eliminating the sensitised response to the true initiating substance. This suggests that the two responses are mediated by different mechanisms although it could be argued that repeated conditioning would account for the pattern observed in some people. Clearly, the involvement of parts of the same neurological system in both mechanisms makes the potential for some degree of cross over more likely.

In summary, although authors have suggested a role for conditioning in MCS, there is not the evidence which supports some other theories. Also, the response pattern for conditioning, in terms of the nature and pattern of the response, is not entirely consistent with that reported by patients with MCS. Therefore, although it cannot be discounted for some people, conditioning does not seem to offer a substantive model.

\section{Psychiatric disorders}

Many authors have concluded that the relatively high incidence of clinical anxiety or depressive states among patients with MCS is evidence that the symptoms are entirely psychogenic. Some also suggest that symptoms are fostered by an iatrogenic "belief" system established by those providing treatment. ${ }^{31}$ Many report high scores on various psychiatric or psychological dimensions. For example, a limited study showed "environmentally ill" subjects to have an excess of personality disorders and traits; depressive and phobic anxiety symptoms; and somatic and hypochondriacal symptoms, suggesting that these patients may have unrecognised emotional problems ${ }^{61}$ Some aspects of conditioning have been combined into a model which suggests that MCS can be considered as a phobic disorder derived from a combination of classical and operant conditioning. ${ }^{30}$ The authors use a small series of three case studies to support this theory. However, many previous authors have drawn on larger data sets to support a psychiatric aetiology for MCS or related diagnoses. In one of the few studies not conducted in the United States, Köppel and Fahron reported on a study of 120 patients presenting to an environmental toxicology service. ${ }^{62}$ Eighty three of the 120 were diagnosed as having some form of psychiatric disorder including somatoform disturbances and 69 had depressive disease. The diagnoses were not necessarily exclusive and the procedures for referral to the service and the diagnostic procedures used are not documented.

Many of the studies of MCS purporting to show a psychosomatic cause, draw this infer- ence from the levels of depression, anxiety, etc among patients. It is not therefore possible to exclude the suggestion that these symptoms are an effect of the MCS rather than causal. Bell et al support this, citing work which shows that up to $45 \%$ of patients with cancer, heart disease, etc are depressed. ${ }^{44}$ Some retrospective assessment of psychiatric state before the onset of MCS has been reported. For example, seven out of 13 subjects diagnosed as environmentally ill (not necessarily MCS) were judged to already have anxiety or depression (with a National Institute of Mental Health diagnostic interview schedule). ${ }^{60}$ In an alternative to retrospective diagnosis based on interviews, Terr reviewed the medical records of a total of 90 patients claiming compensation for work related environmental illness. ${ }^{63}$ Sixty two of these reported "multisystem polysymptomatology". There were 38 diagnoses (not necessarily 38 separate people) of psychiatric disorders before the reported onset of environmental illness. These included depression, anxiety, somatisation disorder, and stress. No indication is given of the numbers for each disorder or of how these related to those with multisystem problems.

Fiedler et al identified 36 subjects classified as either MCS (fulfilling most of the criteria laid down by Cullen ${ }^{12}$ ) or chemical sensitivity (no identifiable initiating exposure). ${ }^{64}$ All would be classified as MCS with the criteria adopted for this review. Compared with a group of 18 normal controls, both groups contained significantly more people with either current or lifetime psychiatric diagnoses - such as depression or anxiety. However, $<50 \%$ of the 36 were diagnosed with such problems, underlining the complexity of attempting to explain the aetiology of MCS.

The pre-existence of psychiatric problems can be used to counter the argument that such problems are an effect of the MCS rather than a cause. However, this could indicate a greater sensitivity to MCS among those susceptible to depression or other psychiatric disorders. "Prior psychiatric symptomatology neither proves a psychogenic aetiology nor disproves an environmental one". ${ }^{1}$

A recent appraisal of the literature on a psychogenic origin for MCS examined 10 articles which reported original data and identified extensive methodological deficiencies in nine. ${ }^{65}$ In particular, eight of the 10 confused association (correlation) with cause, in cross sectional analysis.

Pennebaker documented several personal characteristics relevant to MCS. ${ }^{59}$ For example, women are more likely to report physical symptoms in environments perceived to be stressful. People with chronic anxiety (measured as negative affectivity) are more likely to regard bodily sensations in a negative light and also more likely to report symptoms. The author also documented the increased tendency for people who had undergone trauma to report symptoms, and as reported earlier, discuss various theories to account for this. Although these relations have been documented independently of studies of MCS, they 
can explain some of the reported associations between MCS and psychological factors.

One further paper of particular interest, especially relative to alleged iatrogenic causes and the suggestion that "MCS constitutes a belief and not a disease", ${ }^{1}$ is that published by Dalton, reporting a study in which the subjective impact of an odour was strongly influenced by the perceived hazard it presented.$^{66}$ Subjects given instructions intended to create a hazardous impression were significantly more likely to report that the odour was increasing in strength and offer unsolicited reports of adverse health effects than those given either a positive or a neutral impression.

In conclusion, there seem to be reasonably well documented associations between MCS and psychological characteristics although it is not possible to identify a causal relation from these. Psychogenic theories have been the subject of considerable adverse comment, particularly from the advocates of other theories ${ }^{67}$; however, some authors who support biological explanations also acknowledge the involvement of psychological factors. ${ }^{44}$

\section{General discussion}

CAUSAL THEORIES

The scientific literature on MCS presents a sometimes conflicting and contradictory picture of the mechanisms and causes with growing support for the contention that MCS is not a single, distinct, clinical entity. ${ }^{112168}$

Statistical associations between psychiatric disorders - such as depression and anxietyhave led many to conclude that MCS is a psychogenic disease state although the opposing causal association (MCS causing the depression) is often equally valid. Psychological traits such as shyness $^{50}$ and negative affectivity (related to anxiety), ${ }^{14}$ seem to be related to MCS. Some authors interpret this relation as implying a susceptibility and it is plausible that anxiety or depressive states could play a similar part. There is no doubting the power of psychological suggestibility, and although some authors have chosen to categorise MCS as a belief fostered by those providing the treatment, ${ }^{129}$ others have reviewed such theories and concluded that although tenable in some individual cases, it is highly unlikely that such a mechanism can account for most cases. Similarly, although a psychogenic origin — such as conditioning - may account for some cases, the response pattern of many does not support this as a general diagnosis. Bell et al sound a note of caution about this, stating that the ability to treat clinical symptoms with a psychological approach does not necessarily prove a psychological aetiology. ${ }^{42}$

It is difficult to prove or disprove the suggestion that, in some people, MCS may be wholly psychogenic or represent the manifestation of a psychiatric disease state. There is a tendency to regard this as the default explanation. For example, Bock and Birbaumer specifically state that, after exclusion of toxic and primary immunological and psychiatric causes, it should always be assumed that MCS has a psychological aetiology. ${ }^{69}$ This is presumptuous when other plausible mechanisms require further exploration and tends to make MCS a "diagnosis of exclusion". The distinction between mind and body is also becoming increasingly indistinct. For example, growing evidence for a psychosocial influence in the aetiology of musculoskeletal disorders ${ }^{70}$ shows the difficulties that may emerge in the future in apportioning relative blame to different physical and psychological risk factors. Although some experts maintain that these disorders are wholly of psychological origin others maintain that, although psychological factors may contribute to their development, some physical influences must be involved.

As with the psychological theories, some biologically based theories have attracted support although the evidence is conflicting. Any such mechanism must account for the fact that, with current conventional understanding of toxicology, not all cases have an apparent, discrete precipitating exposure which could evoke a toxicological or immunological response. Also, once MCS is initiated, symptoms are provoked by exposures at concentrations well below any considered as remotely of relevance by mainstream toxicologists. Although beyond the remit of this review, there may be some value in examining putative mechanisms for the apparent efficacy of subtherapeutic doses of homeopathic remedies.

Detailed exposure data for most initiating and triggering exposures are conspicuous by their absence. Both qualitative informationfor example, details of the specific pesticides involved when these are implicated in the onset-and quantitative exposure are not generally available. Curiously, MCS seems to be less common among those populations who might be presumed to be more at risk-for example, those working with chemicalsalthough the data on which this assertion is based would probably not stand up to the more rigorous scrutiny normally afforded to epidemiological studies. Although explanations of variation in individual susceptibility can plausibly account for why some people have MCS and others do not, this does not explain these apparent population differences unless some coincidental self selection is occurring in employment in the chemical industry. It should not be assumed that manufacturing chemicals necessarily results in increased exposure.

Although they have many proponents, explanations based on immunological disorders or deficiencies are not particularly satisfactory. Some studies have shown abnormal immunological characteristics among patients with MCS. It is likely that such people form a different group of subjects, who, although allergic to multiple chemicals, are not genuinely multichemical, multisystem sensitive. The pattern of abnormality is widely variable. This could be partly explained invoking the concept of MCS as various disorders sharing a common pattern of symptoms, although the inconsistency of response would seem to be too broad to even allow groups of response patterns to emerge. As well as the precipitating exposure not necessarily being what would normally be considered excessive (some cases 
of MCS seem to stem from remodelling buildings, drawing parallels with sick building syndrome) the subsequent exposures sufficient to provoke symptoms are considered to be well below those normally regarded as a toxic dose (without invoking theories of dramatically heightened sensitivity).

Some studies have shown that physiological (electroencephalographical) responses can be evoked by chemical exposures below the odour threshold, clearly indicating the presence of other detection mechanisms. ${ }^{49}$ In a different field, Friedman et al showed that pharmaceuticals previously considered not to reach brain tissue because of the blood brain barrier could do so under stressful conditions. ${ }^{71}$ Such evidence provides support for theories - such as that of limbic kindling. Although the research of Friedman et al did not identify the area of the brain involved, the close physical proximity between the areas involved in chemical or odour sensation and those implicated in limbic kindling renders such a mechanism more plausible. Certainly, neither this nor the fact that chemical odours below the odour threshold may have wide ranging effects at concentrations well below those considered necessary to provoke responses in more peripheral tissues can be discounted. The link to stressful situations could also account for the reported associations with trauma, whether or not the chemical is perceived as a significant element in that trauma.

As yet, the invocation of limbic kindling as an explanatory mechanism for MCS is purely hypothetical. It seems to fit most, if not all, of the facts concerning MCS and can be supported by knowledge of the neurophysiology of the brain. Interestingly, no paper has yet been found to offer any contradictory evidence or to discount this as a theory in favour of an alternative. By contrast, most other substantial theories have been countered by authors advocating an alternative hypothesis. However, this or any other biologically based theory has to explain why MCS is not more prevalent among groups with higher exposures-for example, solvent misusers ${ }^{21}$ - and account for the apparent lack of a direct dose-response relation.

The proponents of limbic kindling also accommodate the influence of psychological factors within the theoretical mechanism. The evidence for the suggestibility of responses, both that cited here and in other spheres-such as recalled misuse - and the possible influence of psychological traits-such as depression, shyness, and anxiety-both suggest that psychological factors may modify the response to environmental insults - such as chemical exposure. Indeed, many authors partly explain this by some form of individual susceptibility, on the basis of psychological characteristics.

There is, therefore, a need to consider the interplay of psychological and physical factors as initiators and triggers of a disease process and as components of the underlying mechanisms that will determine the organ systems involved in disease outcome. There is a tendency to classify disease as either physical or psychological, whereas in reality the bounda- ries are less well defined. The amygdala has recently been identified as the focus of the fear response. The fear response is complex, and may be initiated or triggered by both physical (trauma) and psychological (perceived harrassment) stimuli. The outcomes can be physical diseases - for example, cardiovascular - or psychological ill-health-for example, anxietyproviding an example of multiple diverse stimuli producing a multisystem response. The amygdala has also been implicated in chemical sensitivity. As well as a role in determining emotions, it also has connections with the olfactory system and the hypothalamus, which governs a range of physiological response in multiple organ systems. Therefore it is feasible, although at present not objectively testable, that the symptom pattern described within MCS can arise as a result of a process which involves the olfactory system, amygdala, and hypothalamus, with or without the presence of additional psychological stimuli.

\section{DIAGNOSTIC CRITERIA}

In an earlier section of this review, it was stated that the defining diagnostic criteria for the inclusion of MCS studies in this review were deliberately set very broadly. It seems appropriate to conclude by briefly examining these diagnostic criteria; MCS is often criticised because of the essentially response based nature of its diagnostic characteristics. Ashford and Miller include it as an effect based name for heightened reactivity to chemicals. ${ }^{18}$ They also document proposed case definitions and research case definitions for MCS, published by various authors or organisations.

The requirement for the disorder to be acquired in response to a demonstrable or documented environmental exposure is not widely endorsed, with many authors indicating that a proportion of their cases do not necessarily meet this criterion. As Ashford and Miller indicate, earlier exposure of this nature is only a prerequisite under the Cullen criteria. ${ }^{18}$ Despite this, the questionnaire included by these authors assumes such an exposure or exposures, with most questions relating to before or since exposure.

Most authors necessarily specify the involvement of more than one organ system. Similarly, most specify that the symptoms are necessarily evoked by various chemicals. Even the requirement for symptoms to be elicited by very low exposures are not explicit in all texts, although they are generally implied. For example, in their recent text, Ashford and Miller do not specify the level of exposure considered to initiate this process. ${ }^{18}$

Clearly, it is important to exclude other diagnoses. The possibility that a person may have other disorders concurrent with MCS means that care should be taken in excluding such patients from any MCS case definition.

There has been a general tendency to focus on criteria that are required to constitute a diagnosis of MCS. The variability in criteria has contributed to the diversity of opinion on the underlying disease process. In the light of the findings of this review it is perhaps 
appropriate to consider the pathways and physiological processes which would need to be involved to produce the outcomes described for MCS, given the nature of the initiating and triggering stimuli.

\section{Further directions for research}

Further research needs to focus on elucidating the basic epidemiology of MCS and testing specific hypotheses about causes of the disease. Reliable data on the prevalence of MCS are important in determining the scale of controls that would be required to reduce the risk of this condition within susceptible groups.

There is currently a lack of reliable information on the prevalence of MCS. Any population survey would be limited by the lack of knowledge about this condition within the general population, and the variable diagnostic and classification procedures applied by the medical profession. It may be preferable to investigate the incidence of MCS within those industries where exposure to specific groups of chemicals might be expected to produce this condition in a small group of susceptible people. This approach would also allow more reliable measurement of exposures and further exploration of the concept of initiation and triggering. It is likely that epidemiology will help in improving the case definition for MCS.

The other area of emphasis should focus on a better understanding of the mechanisms by which low chemical exposures can produce the range of symptoms described within MCS. Researchers within the United States have already explored the range of research options which are available and these are documented in the scientific literature. Further research may include follow up of previously exposed groups; prospective follow up of populations involved in such incidents as chemical spillages; double blind placebo controlled studies within specially designed medical units; or animal experiments designed to explore the underlying mechanisms which may be associated with chemical sensitivity. There is always the possibility that some other theory, not hitherto applied to MCS - such as health belief models - may also provide a viable explanation for this phenomenon. The concepts of masking and toxic induced lack of tolerance both merit consideration when attempting to explain the variability of response to challenge tests reported in studies of MCS. Finally, it seems that there is a range of individual susceptibility to the effects of chemicals. This should be considered in the design and interpretation of future research into MCS.

\section{Conclusions}

There is evidence to suggest that in some people exposure to chemicals can initiate a clinical response to subsequent exposures to very low doses of that chemical and structurally unrelated chemicals. It is likely however, that this accounts for only a small percentage of people labelled as having MCS. The evidence is convincing if it takes account of the pattern of symptoms and mode of acquisition of the illness reported by this subgroup. Also, case history evidence of the progressive negative impact on lifestyle, without apparent benefit to the individual person offers further support.

Objective evidence is, however, lacking. There is a paucity of reliable exposure data, no agreed test battery, and the most plausible disease models are based on animal studies with no ready means of confirming the hypotheses in humans. Also, many researchers try to identify a mechanism or abnormality within one organ system rather than seeking an explanation for the multisystem response in MCS.

As MCS does not fit established knowledge regarding disease processes, many people reach the conclusion that MCS must be psychological in origin. The power of the psyche should never be underestimated and there may be people for whom the description of MCS as a belief state may be apt.

Among the theories suggesting a physical explanation for MCS, that of limbic kindling and its associated processes cannot as yet be excluded. This theory provides an explanation for a sensitisation process and allows for the modifying effect of coexisting or preexisting psychological factors. For sensitisation to occur, both an initiating event and subsequent triggering events are required. It is plausible that components of unrelated chemicals could share certain carbon chain links with similar surface activity able to trigger a response in a previously sensitised person (where the initiating event occurred with another chemical). Recent research has also suggested that certain chemicals are able to directly penetrate areas of the brain and to exert an effect at doses much lower than previously considered possible.

At present this remains a theory with no direct experimental evidence. To confirm or refute this theory, more research is required into the role of the limbic system in humans. This research should be carried out according to agreed protocols, with validated and objective test methods. It is also likely that by obtaining more reliable scientific data, the underlying processes, which are involved in response to both initiating and triggering stimuli, can be better delineated. This would reduce the reliance now placed on definitions of MCS in the diagnosis of individual cases.

Current evidence does not prove beyond reasonable doubt, in all cases, the existence of a condition fitting the review criteria for MCS, but available evidence in some people cannot be ignored and warrants further investigation.

This work was commissioned and sponsored by the United Kingdom Health and Safety Executive. However, the ideas and opinions expressed are those of the authors.

1 Terr AI. Multiple chemical sensitivities: immunologic critique of clinical ecology theories and practice. Occup Med 1987;2:683-94.

2 Samet J, Davis DL. Introduction. Multiple chemical sensitivities: a workshop. Washington, DC: National Academic Press, 1992:1-4. In: Mitchell FL, ed. Multiple chemical sensitivity: a scientific overview. Washington, DC: US Department of Health and Human Services, 1995 .

3 Smith F. Charles Darwin's ill health. Fournal of the History of Biology 1990;23:443-59.

4 Smith F. Charles Darwin's health problems: the allergy hypothesis. Fournal of the History of Biology 1992;25:285-306.

hypothesis. Fournal of the History of Biology 1992;25:285-306.
Levin AS, Byers VS. Environmental illness: a disorder of immune disregulation. Occup Med 1987;2:669-81. 
6 Rea WJ, Johnson AR, Ross GH, et al. Considerations for the diagnosis of chemical sensitivity. Multiple chemical demic Press, 1992;169-92. In: Mitchell FL, ed. Multiple demic Press, 1992;169-92. In: Mitchell FL, ed. Multiple chemical sensitivity: a scientific overview. Washington,

7 Schottenfeld RS. Workers with multiple chemical sensitivities: a psychiatric approach to diagnosis and treatment. Occup Med 1987;2:739-53.

8 Staudenmayer H, Selner ME, Selner JC. Adult sequelae of childhood abuse presenting as environmental illness. Ann Allergy 1993b;71:538-46.

9 Kroll-Smith S, Ladd AE. Environmental illness and biomedicine: anomalies, exemplars, and the politics of the body. Sociological Spectrum 1993;13:7-33.

10 Miller CS. White paper: chemical sensitivity: history and phenomenology. Toxicol Ind Health 1994;10:253-76.

11 Mooser SB. The epidemiology of multiple chemical sensitivities (MCS) Occup Med 1987;2:663-8.

12 Cullen MR. The worker with multiple chemical sensitivities: an overview. Occup Med 1987;2:655-61.

13 Ziem G, McTamney J. Profile of patients with chemical injury and sensitivity. Environ Health Perspect 1997;105:417-36.

14 Davidoff AL, Keyl PM. Symptoms and health status in individuals with multiple chemical sensitivities syndrome from four reported sensitising exposures and a general population comparison group. Arch Environ Health 1996;51:201-13.

15 Meggs WJ, Cleveland CH. Rhinolaryngoscopic examination of patients with the multiple chemical sensitivity syndrome. Arch Environ Health 1993;48:14-18.

16 Hileman B. Special report: multiple chemical sensitivity Chemical and Environmental News 22 July 1991:26-42.

17 Cullen MR, Pace PE, Redlich CA. The experience of the Yale occupational and environmental medicine clinics with multiple chemical sensitivities, 1986-91. Toxicol Ind Health 1992;8;15-19

18 Ashford NA, Miller CS. Chemical exposures, 2nd ed. New York: Van Nostrand Reinhold, 1998.

19 Cone JE, Harrison R, Reiter R. Patients with multiple chemical sensitivities: clinical diagnostic subsets among an occupation $721-38$.

20 Grimmer A, Géraut C, Dupas D, et al. Syndrome d'intolérance aux odeurs chimiques: á propos de 30 cas. Archives des Maladies Professionnelles et de Médicine du Travail 1995;56:149-50

21 Sparks PJ, Daniell W, Black DW, et al. Multiple chemica sensitivity syndrome: a clinical perspective I. Case definition, theories of pathogenesis and research needs. $7 \mathrm{Occup}$ Med 1994;36:718-30.

22 Berkson J. Patient statement: a canary's tale. Toxicol Ind Health 1994:10:323-6.

23 Sagalowsky AI, Wilson JD. Hyperplasia and carcinoma of the prostate. In: Wilson JD, Braunwald E, Isselbacher KJ, et al, eds. Principles of Internal Medicine. Vol 2. New York:

24 Miller CS, Ashford NA. Possible mechanisms for multiple chemical sensitivity: the limbic system and others. Multiple chemical sensitivities: a workshop. Washington, DC: chemical sensitivities: a workshop. Washington, DC: National Academic Press, 1992:139-150. In: Mitchell FL, ed. Multiple chemical sensitivity: a scientific overview. Washington, D

25 Cone JE, Sult TA. Acquired intolerance to solvents following pesticide/solvent exposure in a building: a new group of workers at risk for multiple chemical sensitivities? Toxicol Ind Health 1992;8:29-39.

26 Simon GE. Epidemic multiple chemical sensitivity in an industrial setting. Toxicol Ind Health 1992;8;41-6.

27 Lax MB, Henneberger PK. Patients with multiple chemical sensitivities in an occupational health clinic: presentation and follow up. Arch Environ Health 1995;50:425-31.

28 Staudenmayer H, Selner JC, Buhr MP. Double-blind provocation chamber challenges in 20 patients presenting with "multiple chemical sensitivity". Regul Toxicol Pharmacol 1993;18:44-53.

29 Brodsky CM. Multiple chemical sensitivities and other environmental illness: a psychiatrists view. Occup $\mathrm{Med}$ environmental it

30 Guglielmi RS, Cox DJ, Spyker DA. Behavioural treatment of phobic avoidance in multiple chemical sensitivity. $\mathcal{F}$ Behav Ther Exp Psychiatry 1994;25:197-209.

31 Black DW. Iatrogenic (physician-induced) hypochondriasis: four patient examples of chemical sensitivity. Psychosomatics 1995;37:390-3

32 Rea WJ, Bell IR, Suits CW, et al. Food and chemical susceptibility after environmental chemical overexposure: case histories. Ann Allergy 1978;41:101-10.

33 Rea WJ, Liang H. Effects of pesticides on the immune system. Fournal of Nutritional Medicine 1991;2:399-410.

34 Albright JF, Goldstein RA. Is there evidence of an immunologic basis for multiple chemical sensitivity? Toxicol Ind Health 1992;8:215-19.

35 Terr AI. Immunological issues in "multiple chemical sensitivities". Regul Toxicol Pharmacol 1993;18:54-60.

36 Wolf C. Multiple chemical sensitivities: is there a scientific basis? Int Arch Occup Environ Health 1994;66:213-16.

37 Kipen H, Fiedler N, Maccia C, et al. Immunologic evaluation of chemically sensitive patients. International fournal of Occupational Medicine and Toxicology 1995;4: $467-77$.

38 Doty RL, Deems DA, Frye RE, et al. Olfactory sensitivity, nasal resistance and autonomic function in patients with multiple chemical sensitivities. Arch Otolaryngol Head Neck Surg 1988;114:1422-7.

39 Bascom R. Multiple chemical sensitivity: a respiratory disorder? Toxicol Ind Health 1992;8:221-8.

40 Meggs WJ. Neurogenic inflammation and sensitivity to environmental chemicals. Environ Health Perspect 1993;101 234-8

41 Meggs WJ, Dunn KA, Bloch RM, et al. Prevalence and nature of allergy and chemical sensitivity in a general population. Arch Environ Health 1996;51:275-82.

42 Meggs WJ. Neurogenic switching: a hypothesis for a mechanism for shifting the site of inflammation in allergy $54-6$.

43 Antelman SM. Time-dependent sensitisation in animals: a possible model of multiple chemical sensitivity in humans. Toxicol Ind Health 1994;10:335-42.

44 Bell IR, Miller CS, Schwartz GE. An olfactory-limbic model of multiple chemical sensitivity syndrome: possible relaionships to kindling and affective disorders. Biol Psychiatry 1992:32:218-42.

45 Adamec R. Modelling anxiety disorders following chemical exposures. Toxicol Ind Health 1994;10:391-420.

6 Bell IR, Schwartz GE, Peterson JM, et al. Possible timedependent sensitisation to xenobiotics: self-reported illness from chemical odours, foods, and opiate drugs in an older adult population. Arch Environ Health 1993;48:315-27.

47 Corrigan FM, MacDonald S, Brown A, et al. Neurasthenic fatigue, chemical sensitivity and GABAa receptor toxins. Med Hypotheses 1994;43:195-200.

48 Heuser G, Mena I, Alamos F. Neurospect findings in patients exposed to neurotoxic chemicals. Toxicol Ind Health 1994;10:561-71.

49 Lorig TS. EEG and ERP studies of low-level odour exposure in normal subjects. Toxicol Ind Health 1994;10: 579-86.

50 Bell IR, Hardin EE, Baldwin CM, et al. Increased limbic system symptomatology and sensitisability of young adults with chemical and noise sensitivities. Environ Res 1995;70: 84-97.

51 Bell IR. Clinically relevant EEG studies and psychophysiological findings: possible neural mechanisms for multiple chemical sensitivities. Toxicology 1996;111:101-17.

52 Carlson NR. Physiology of behaviour, 4th ed. Boston: Allyn and Bacon, 1977

53 Bell IR, Schwartz GE, Baldwin CM, et al. Individual differences in neural sensitisation and the role of context in illness from low-level environmental chemical exposures. Environ Health Perspect 1997a;105:457-66.

54 Bell IR, Rossi III J, Gilbert ME, et al. Testing the neural sensitisation and kindling hypothesis for illness from low levels of environmental chemicals. Environ Health Perspect 1997; 105:539-47.

55 Miller CS. Toxicant-induced loss of tolerance-an emerging theory of disease? Environ Health Perspect 1997;105:44553

56 Miller CS, Ashford NA, Doty R, et al. Empirical approaches for the investigation of toxicant-induced loss of tolerance. Environ Health Perspect 1997;105:515-19.

57 Daniell WE, Stockbridge HL, Labbe RF, et al. Environmental chemical exposures and disturbances of heme synthesis. Environ Health Perspect 1997;105:37-53.

58 Donnay A. Facts on multiple chemical sensitivity. MCS referral and resources 1997;6:1-4.

59 Pennebaker JW. Psychological bases of symptom reporting: perceptual and emotional aspects of chemical sensitivity. Toxicol Ind Health 1994;10:497-511.

60 Simon GE, Katon WJ, Sparks PJ. Allergic to life: psychological factors in environmental illness. Am $\mathcal{f}$ Psychiatry 1990;147:901-6.

61 Black DW, Rathe A, Godstein RB. Measures of distress in 26 environmentally ill subjects. Psychosomatics 1993;34:131-8.

62 Köppel C, Fahron G. Toxicological and neuropsychological findings in patients presenting to an environmental toxicology service. F Clin Toxicol 1995;33:625-9.

63 Terr AI. Clinical ecology in the workplace. $f$ Occup Med 1989;31:257-61.

64 Fiedler N, Kipen HM, DeLuca J, et al. A controlled comparison of multiple chemical sensitivities and chronic fatigue syndrome. Psychosom Med 1996;58:38-49.

65 Davidoff AL, Fogarty L. Psychogenic origins of multiple chemical sensitivities syndrome: a critical review of the research literature. Arch Environ Health 1994;49:316-25.

66 Dalton P. Odor perception and beliefs about risk. Chem Senses 1996;21:447-58

67 Anthony H, Birtwistle S, Eaton K, et al. Environmental medicine in clinical practice. Southampton: BSAENM Publications, 1997.

68 Ashford NA, Miller CS. Low-level chemical sensitivity: current perspectives. Int Arch Occup Environ Health 1996;68: 367-76.

69 Bock KW, Birbaumer N. MCS (multiple chemical sensitivity): cooperation between toxicology and psychology may facilitate solutions of the problems: commentary. Hum Exp Toxicol 1997;16:481-4.

70 Hanson M, Graveling R, Donnan P. Investigation into the factors associated with symptoms of ULDs in keyboard users. In: Robertson SA, ed. Contemporary ergonomics 1997. users. In: Robertson SA, ed. Contemporary ergo

71 Friedman A, Kaufer D, Shemer J, et al. Pyridostigamine brain penetration under stress enhances neuronal excitability and induces early immediate transcriptional response. Nat Med 1996;2:1382-5. 